University of Wollongong

Research Online

Faculty of Engineering and Information

Faculty of Engineering and Information

Sciences - Papers: Part A

Sciences

$1-1-2010$

Dynamic response analysis of the rotating blade of horizontal axis wind turbine

Xiong Liu

University of Wollongong, xiong@uow.edu.au

Xianmin Zhang

South China University of Technology

Gangqiang Li

Shantou University

Yan Chen

Shantou University

Zhiquan Ye

Shantou University

Follow this and additional works at: https://ro.uow.edu.au/eispapers

Part of the Engineering Commons, and the Science and Technology Studies Commons

Research Online is the open access institutional repository for the University of Wollongong. For further information contact the UOW Library: research-pubs@uow.edu.au 


\title{
Dynamic response analysis of the rotating blade of horizontal axis wind turbine
}

\begin{abstract}
This paper presents a dynamic response analysis of the blade of horizontal axis wind turbines using finite element method. The blade is treated as a cantilever and modeled with two-node beam element. The blade element-momentum theory is applied to calculate the aerodynamic loads. Dynamic inflow and dynamic stall are taken into account to reflect the transient aerodynamics. The centrifugal stiffening is introduced to consider the restoring effects of centrifugal force. An aerodynamic damping model is presented for calculating the overall damping ratio instantaneously during time-domain simulation. The structural dynamic equation is solved using Newmark method and the overall dynamic response of the blade is obtained based on the modal superposition principle. Applying the proposed method, the power production load case of a $1.0 \mathrm{MW}$ wind turbine operating in turbulent wind field is simulated. The simulation results indicate that the blades of large-scale horizontal axis wind turbines undergo significant vibration and deflection during operation, and the centrifugal stiffening and aerodynamic damping both considerably affect the structural response of the blade.
\end{abstract}

\section{Keywords}

rotating, blade, horizontal, analysis, axis, turbine, wind, dynamic, response

Disciplines

Engineering | Science and Technology Studies

\section{Publication Details}

Liu, X., Zhang, X., Li, G., Chen, Y. \& Ye, Z. (2010). Dynamic response analysis of the rotating blade of horizontal axis wind turbine. Wind Engineering, 34 (5), 543-560. 


\section{Dynamic Response Analysis of the Rotating Blade of Horizontal Axis Wind Turbine}

by

Liu Xiong, Zhang Xianmin, Li Gangqiang, Chen Yan, Ye Zhiquan

\section{REPRINTED FROM \\ WIND ENGINEERING}

Volume 34, No. 5, 2010 


\title{
Dynamic Response Analysis of the Rotating Blade of Horizontal Axis Wind Turbine
}

\author{
Liu Xiong*,+, Zhang Xianmin*, Li Gangqiang ${ }^{+}$, Chen Yan+, Ye Zhiquan ${ }^{+}$ \\ *College of Mechanical Engineering, South China University of Technology, Guangzhou, \\ China, 5 I 064 I \\ +Institute of Energy Science, Shantou University, Shantou, China, 5 I5063 \\ E-mail: Ix@stu.edu.cn
}

\begin{abstract}
This paper presents a dynamic response analysis of the blade of horizontal axis wind turbines using finite element method. The blade is treated as a cantilever and modeled with two-node beam element. The blade element-momentum theory is applied to calculate the aerodynamic loads. Dynamic inflow and dynamic stall are taken into account to reflect the transient aerodynamics. The centrifugal stiffening is introduced to consider the restoring effects of centrifugal force. An aerodynamic damping model is presented for calculating the overall damping ratio instantaneously during time-domain simulation. The structural dynamic equation is solved using Newmark method and the overall dynamic response of the blade is obtained based on the modal superposition principle. Applying the proposed method, the power production load case of a $1.0 \mathrm{MW}$ wind turbine operating in turbulent wind field is simulated. The simulation results indicate that the blades of large-scale horizontal axis wind turbines undergo significant vibration and deflection during operation, and the centrifugal stiffening and aerodynamic damping both considerably affect the structural response of the blade.
\end{abstract}

Keywords: Wind turbine; Dynamic response; Centrifugal stiffening; Aerodynamic damping

\section{INTRODUCTION}

The development of increasingly large-scale horizontal axis wind turbines (HAWTs) is leading to long blade designs. With the application of GRP composite material, the blade is becoming much more flexible than ever before. The consequence is large blade deflections and vibrations that require special design and computational considerations regarding loads, displacement, blade-tower interaction, etc. The deflection and vibration not only lead to additional stresses which influence the blade strength, but also affect the aerodynamic characteristics of the blade, resulting in the change of the aerodynamic load distribution on the blade. Therefore in the wind turbine design procedure, it is essential to study the structural dynamics of the blade and understand its dynamic response subjected to the timevarying load.

In the early days of the industry, wind turbine design was undertaken on the basis of quasistatic aerodynamic calculations with the effects of structural dynamics either ignored completely or included through the use of estimated dynamic magnification factors [1,2]. From the late 1970s, research workers began to consider more reliable methods of dynamic 
analysis for wind turbine, and three basic approaches were considered: finite element method (FEM) [3-5], modal analysis method (MAM) [6-8] and multi-body system dynamics (MBS) [9-11]. Generally, because FEM has the maximum degree of freedom requirement and high computational cost, it has not proven itself to be suitable for modeling the whole wind turbine system. But as FEM is a high precision method, which can accurately simulate the mechanical behavior of the flexible structures of the wind turbine, it is often applied to analyze the main flexible parts of the turbine-blade and tower-with beam elements. MAM, such as mode superposition method or assumed modes approach, can reflect the dynamic behavior of the turbine with relatively fewer degrees of freedom. But this method is usually used in the preliminary design stage, because it is not suitable for modeling the nonlinear behaviors such as large deflections of the flexible structures. MBS has the most complexity in model setup, so it is often implemented by coupling an aeroelastic program and professional MBS software such as ADAMS or SIMPACK.

Because of the natural aerodynamic characteristics of the blade airfoils, the vibration of the blade is not only affected by structural damping, but also aerodynamic damping. Researches of Risø laboratory [12] indicate that, before stall the aerodynamic damping is the main resistance of the blade vibration, but after stall the aerodynamic damping can become negative, may lead to the damage of the blade. Thus the study of aerodynamic damping characteristics of the wind turbine blade during dynamic process attracts more and more attentions $[13,14]$.

Aimed to implement a dynamic response analysis method which is suitable for engineering application, this paper applies FEM to model the HAWT blade. The natural frequencies and mode shapes in the out-of-plane and in-plane directions are obtained with the consideration of centrifugal stiffening. The aerodynamic damping is also taken into account and its time series is calculated instantaneously during the time-domain simulation. The structural dynamic equation is resolved by applying Newmark method and the overall dynamic response of the blade is obtained using modal superposition principle. Applying the proposed method, the dynamic response of the blade of a 1.0 MW HAWT operating in turbulent wind field is calculated and the influences of centrifugal stiffening and aerodynamic damping on the structure response are analyzed.

\section{FINITE ELEMENT DYNAMIC EQUATION OF THE ROTATING BLADE}

\section{I. Blade Finite Element Model}

Because the aspect ratio of the horizontal axis wind turbine blade is very large, the blade can be treated as a cantilever and applied two-node beam element to discrete it. As shown in figure 1, assuming $l$ is the element length, $\rho_{e}$ is the element density, $\boldsymbol{N}$ is the hermitian interpolation function matrix, $E$ is the elastic modulus, $I$ is the sectional inertia moment, the mass matrix $\boldsymbol{M}_{e}$ and stiffness matrix $\boldsymbol{K}_{e t}$ for each element can be obtained as [15]

$$
\begin{gathered}
\boldsymbol{M}_{e}=\int \rho_{e} \boldsymbol{N}^{\mathrm{T}} \boldsymbol{N} \mathrm{d} v \\
\boldsymbol{K}_{e t}=\int_{0}^{l} E I\left(\frac{\mathrm{d}^{2} \boldsymbol{N}}{\mathrm{d} x^{2}}\right)^{\mathrm{T}}\left(\frac{\mathrm{d}^{2} \boldsymbol{N}}{\mathrm{d} x^{2}}\right) \mathrm{d} x
\end{gathered}
$$

where $\mathrm{d} v=A d x, A$ is the element sectional area.

The hermitian interpolation function matrix $\boldsymbol{N}$ is obtained as [16]

$$
\boldsymbol{N}=\left[N_{1}(x) \quad N_{2}(x) \quad N_{3}(x) \quad N_{4}(x)\right]
$$




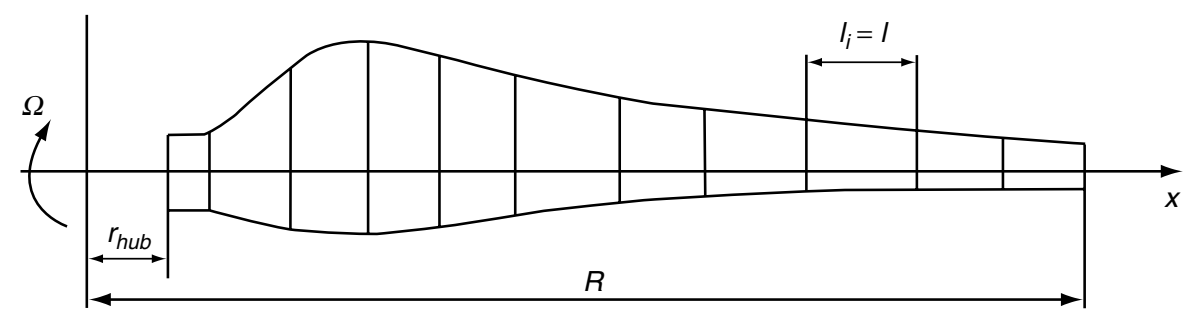

Figure 1: FEM model of the wind turbine blade.

where $N_{i}(x)$ are cubic hermitian polynomials which may be expressed as

$$
N_{1}(x)=1-3\left(\frac{x}{l}\right)^{2}+2\left(\frac{x}{l}\right)^{3}, \quad N_{2}(x)=3\left(\frac{x}{l}\right)^{2}-2\left(\frac{x}{l}\right)^{3}, \quad N_{3}(x)=x\left(1-\frac{x}{l}\right)^{2}, \quad N_{4}(x)=\frac{x^{2}}{l}\left(\frac{x}{l}-1\right)
$$

From equation (1) and (2), we can get

$$
\begin{gathered}
\boldsymbol{M}_{e}=\frac{\rho_{e} l A}{420}\left(\begin{array}{cccc}
156 & 54 & 22 l & -13 l \\
54 & 156 & 13 l & -22 l \\
22 l & 13 l & 4 l^{2} & -3 l^{2} \\
-13 l & -22 l & -3 l^{2} & 4 l^{2}
\end{array},\right. \\
\boldsymbol{K}_{e t}=\frac{2 E I}{l^{3}}\left(\begin{array}{cccc}
6 & -6 & 3 l & 3 l \\
-6 & 6 & -3 l & -3 l \\
3 l & -3 l & 2 l^{2} & l^{2} \\
3 l & -3 l & l^{2} & 2 l^{2}
\end{array}\right)
\end{gathered}
$$

The sectional stiffness of the blade is usually given in flapwise and edgewise directions, but in reality the dynamic responses in out-of-plane and in-plane directions are required. Thus firstly it is needed to transform the stiffness to out-of-plane and in-plane directions with the consideration of sectional twist angle. As shown in figure 2, assuming $o$ is the sectional centroid, the sectional bending stiffness in out-of-plane and in-plane directions can be given as

$$
\begin{aligned}
& E I_{X}=E I_{F} \cos ^{2} \theta_{b}+E I_{c} \sin ^{2} \theta_{b} \\
& E I_{Y}=E I_{F} \sin ^{2} \theta_{b}+E I_{c} \cos ^{2} \theta_{b}
\end{aligned}
$$

where $I_{F}$ is the sectional inertia moment about axis $\zeta, I_{F}=\int_{A} \eta^{2} d A, I_{C}$ is the sectional inertia moment about axis $\eta, I_{c}=\int_{A} \zeta^{2} d A, \theta_{b}$ is the sectional twist angle.

When a rotating blade deflects either in its plane of rotation or perpendicular to it, the centrifugal force on each blade element exerts a restoring force which has the effect of stiffening the blade and thereby increasing the natural frequency compared with the stationary value. In order to take account of the effects of centrifugal loads, the geometric stiffness matrix is needed to be introduced. By using hermitian interpolation functions, the consistent geometric stiffness matrix is obtained as [16]

$$
\boldsymbol{K}_{e g}=\int_{0}^{l} \boldsymbol{N}^{\prime \mathrm{T}} N(x) \boldsymbol{N}^{\prime} d x
$$

where $\boldsymbol{N}^{\prime}$ is the first derivative of $\boldsymbol{N}$ with respect to $x, N(x)$ is the distributed centrifugal force. 


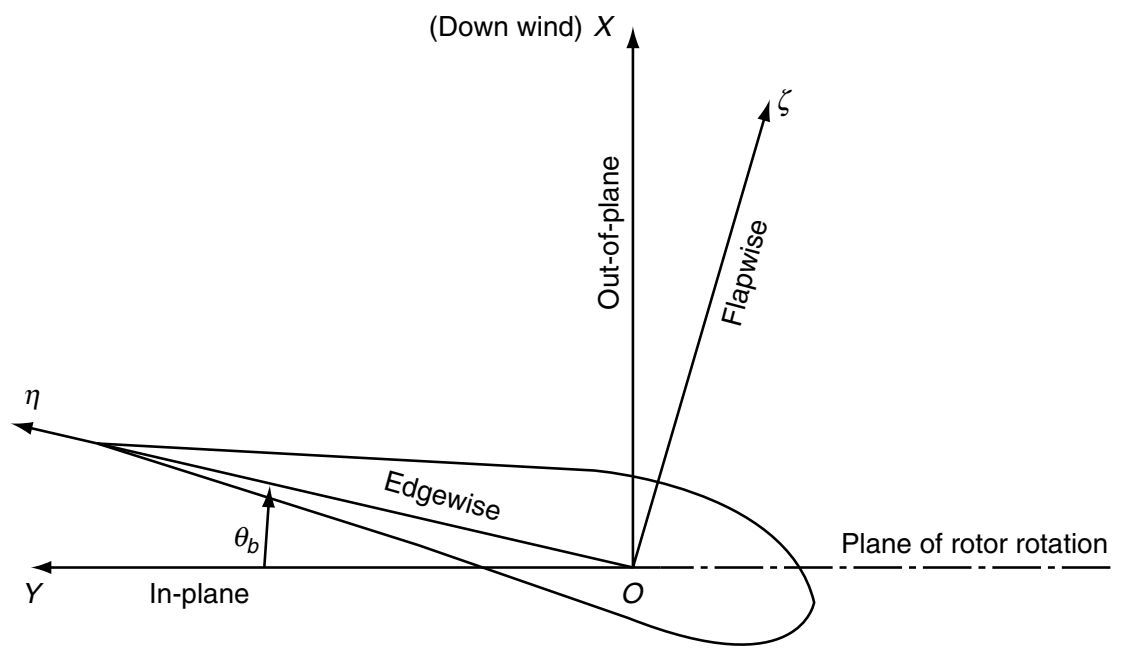

Figure 2: Coordinate system for transformation of the sectional stiffness.

In the specific case where the centrifugal force is constant over the length of the element, the consistent geometric stiffness matrix is

$$
\boldsymbol{K}_{e g}=\frac{N_{g}}{30 l}\left(\begin{array}{rrrc}
36 & -36 & 3 l & 3 l \\
-36 & 36 & -3 l & -3 l \\
3 l & -3 l & 4 l^{2} & -l^{2} \\
3 l & -3 l & -l^{2} & 4 l^{2}
\end{array}\right)
$$

where $N_{g}$ is the centrifugal force acting on the element.

The combined stiffness matrix is obtained by adding the consistent geometric stiffness matrix to the elastic stiffness matrix, which includes both elastic and geometric effects [17].

$$
\boldsymbol{K}_{e}=\boldsymbol{K}_{e t}+\boldsymbol{K}_{e g}
$$

\subsection{Equation of Motion of the Rotating Blade}

Assembling element mass matrix and stiffness matrix from the known blade structure geometric parameters, the blade MDOF (Multi-Degree of Freedom) equation of motion subjected to the time-varying load can be obtained as

$$
\boldsymbol{M} \ddot{\boldsymbol{x}}(t)+\boldsymbol{C} \dot{\boldsymbol{x}}(t)+\boldsymbol{K} \boldsymbol{x}(t)=\boldsymbol{P}(t)
$$

where $\boldsymbol{M}$ is the system mass matrix, $\boldsymbol{C}$ is the system damping matrix, $\boldsymbol{K}$ is the system stiffness matrix, $\boldsymbol{P}(t)$ is the time-varying load matrix, $\ddot{\boldsymbol{x}}(t), \dot{\boldsymbol{x}}(t), \boldsymbol{x}(t)$ are the acceleration, velocity and displacement vectors of the finite element nodes respectively.

Considering equation (10), if $\boldsymbol{P}(t)=0$, the blade is in the status of free vibration. The damping effect is usually neglected and eigenvalues can be calculated using equation

$$
\left(\boldsymbol{K}-\omega^{2} \boldsymbol{M}\right) \boldsymbol{\Phi}=0
$$

Then the structure vibration mode matrix $\Phi=\left(\begin{array}{llll}\phi_{1} & \phi_{2} & \cdots & \phi_{n}\end{array}\right)$ and natural frequencies $\omega_{n}(n=1,2, \ldots, N)$ can be obtained.

\section{ANALYSIS OF BLADE LOADING}

The time-varying loads of a rotating blade mainly include aerodynamic force, centrifugal force and gravity. To account for blade loads and deflections, a coordinate system is defined in figure $3: Z B$ is radially along blade axis; $X B$ is perpendicular to $Z B$ and pointing towards the 


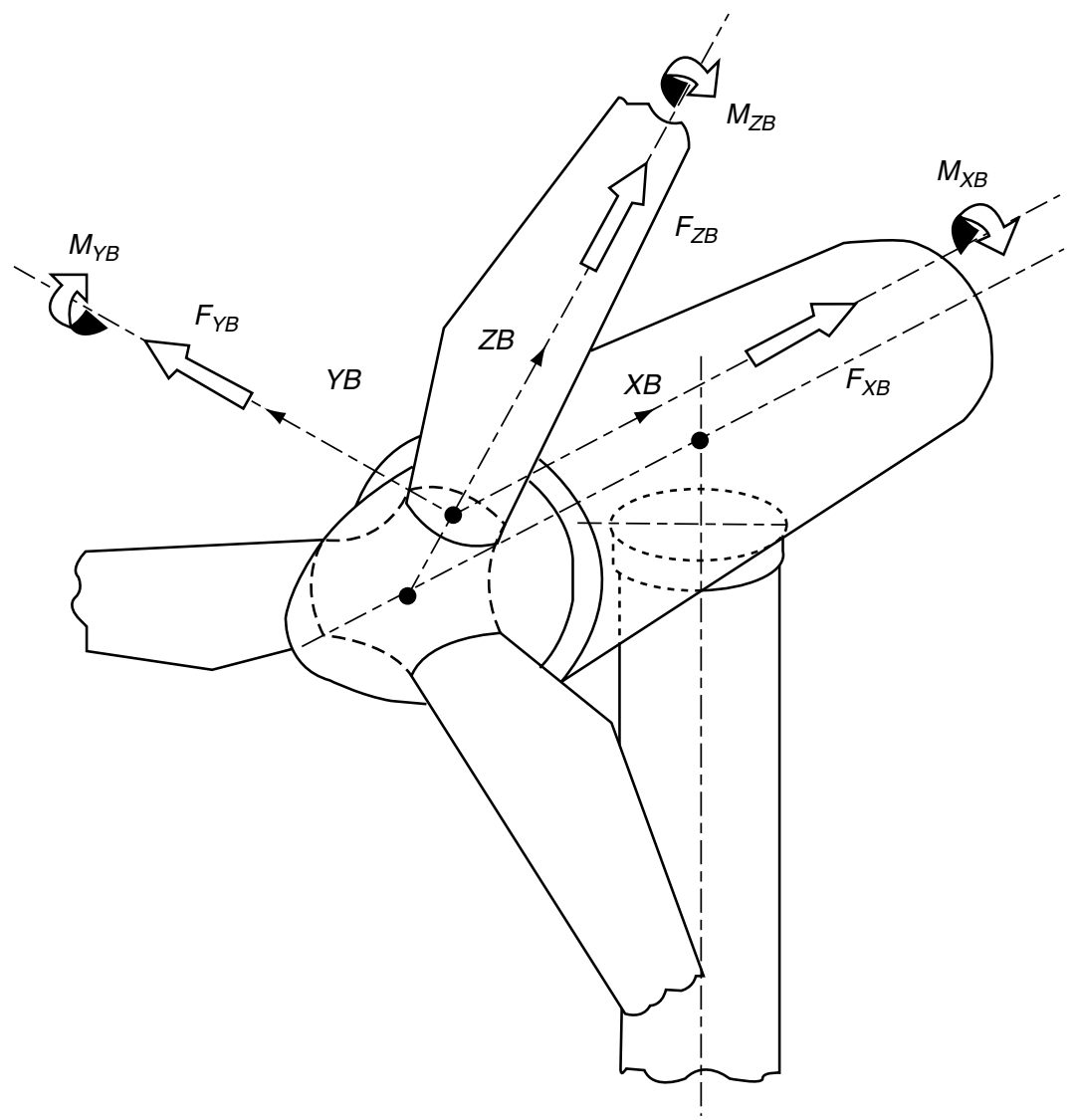

Figure 3: Coordinate system for blade loads and deflections.

tower for an upwind turbine; $Y B$ is perpendicular to blade axis and shaft axis, to give a righthanded coordinate system independent of direction of rotation and rotor location upwind of the tower.

\section{I. Aerodynamic Loads}

Aerodynamic loads of the wind turbine blade operating in steady conditions can be solved by blade element-momentum theory [2,18]. But in dynamic conditions, the dynamic inflow and dynamic stall must be taken into account to correctly reflect the influences caused by wind turbulence, yawing, rotational speed regulation and pitch regulation.

When operating in steady wind, according to blade element-momentum theory, the normal force and tangential force per unit length of the blade are obtained as

$$
\begin{gathered}
F_{X B a}=\frac{1}{2} \rho W^{2} c\left(C_{L} \cos \varphi+C_{D} \sin \varphi\right) \\
F_{Y B a}=-\frac{1}{2} \rho W^{2} c\left(C_{L} \sin \varphi-C_{D} \cos \varphi\right)
\end{gathered}
$$

where $C_{L}, C_{D}$ are the sectional lift coefficient and drag coefficient respectively, $W$ is the sectional relative velocity of airflow, $\varphi$ is the sectional inflow angle, $\rho$ is the air density, $c$ is the sectional chord length.

The momentum theory assumes that the induced velocity flow field reacts instantaneously to the changes in blade loading. In reality this treatment is not strictly correct. The changes in blade loading change the vorticity that is trailed into the rotor wake and the 
full effect of these changes takes a finite time to change the induced flow field [6]. The dynamics associated with this process is commonly referred to as "dynamic inflow".

The study of dynamic inflow was initiated nearly 40 years ago in the context of helicopter aerodynamics. In brief, the theory provides a means of describing the dynamic dependence of the induced flow field at the rotor upon the loading that it experiences. The dynamic inflow model used in this paper is based on the work of Pitt and Peters [19] which has received substantial validation in the helicopter field. The Pitt and Peters model was originally developed for an actuator disk with assumptions made concerning the distribution of inflow across the disc. In this paper the model is applied at blade element or actuator annuli level since this avoids any assumptions about the distribution of inflow across the disc. Simply the thrust coefficient equation of momentum theory is amended as [20]

$$
C_{T}=4 a(1-a)+\frac{8 r}{\pi U} \dot{a}
$$

where $C_{T}$ is the thrust coefficient, $a$ is the axial flow induction factor, $r$ is the radius of actuator annuli, $U$ is the upstream wind velocity.

Equation (14) can therefore be used to replace the blade element-momentum theory equation for the calculation of axial inflow. The equation is integrated at each time step to give time dependent values of inflow for each blade element on the blade. The tangential inflow is obtained in the usual manner and so depends on the time dependent axial value. It is evident that the equation introduces a time lag into the calculation of inflow which is dependent on the radial station.

When airfoils are undergoing unsteady motion, another phenomenon which considerably influences aerodynamic loading is dynamic stall. In this paper, the Beddoes-Leishman (B-L) dynamic stall model [21] is adopted to take account of unsteady behavior of airfoils. The model used within this paper utilizes the following elements of the original B-L model to calculate the unsteady lift coefficient [6, 22-23]: 1) the indicial response functions for the modeling of attached flow; 2) the time lagged Kirchoff formulation for the modeling of trailing edge separation and vortex lift.

Indicial response produces the normal force coefficient $C_{N}$ as a function of time for a step change in angle of attack. The increment in $C_{N}$ due to a step change in angle of attack $\Delta \alpha$ is broken into two components, a non-circulatory component $C_{N}^{I}$, and a circulatory component $C_{N}^{C}$, given as:

$$
\begin{aligned}
\Delta C_{N}^{C} & =C_{N \alpha} \phi_{\alpha}^{C} \Delta \alpha \\
\Delta C_{N}^{I} & =\frac{4}{M} \phi_{\alpha}^{I} \Delta \alpha
\end{aligned}
$$

where $C_{N \alpha}$ is the normal force coefficient curve slope, $M$ is the Mach number, $\phi_{\alpha}^{C}$ is the circulatory indicial function, and $\phi_{\alpha}^{I}$ is the non-circulatory indicial function. In the B-L model the chord wise force coefficient $C_{C}$ response is based on the circulatory component of $C_{N}$. The airfoil attached flow response due to a general angle of attack history is calculated from the superposition of individual indicial responses for each step.

The calculated attached flow response is then modified based on the position of the effective flow separation point on the low pressure side of the airfoil. Flow separation from the airfoil results in a loss of circulation about the airfoil, reducing aerodynamic coefficients from the attached flow values. The separation point is given by $f=x / c$, where $x$ is the point of flow 
separation measured from the leading edge, and $c$ is the airfoil chord length. An approximation to Kirchoff theory used by B-L relates $C_{N}$ to the separation point given as:

$$
C_{N}=C_{N \alpha}\left(\alpha-\alpha_{0}\right)\left(\frac{1+\sqrt{f}}{2}\right)^{2}
$$

where $\alpha$ is the angle of attack, and $\alpha_{0}$ is the zero-lift angle of attack.

The static effective separation point is calculated from static $C_{N}$ data by solving equation (17). The effective separation point versus angle of attack is then curve fit using an exponential function. In the B-L model an empirically derived first order lag is applied to the movement of the effective separation point to account for the time lag in movement of the separation point during unsteady conditions.

The final main component of the model represents the vortex build-up and shedding that occurs during dynamic stall. The vortex lift contribution is empirically modeled as an excess circulation in the vicinity of the airfoil. The magnitude of the increase in lift is based on the difference between the attached flow $C_{N}$, and the $C_{N}$ value obtained from the Kirchoff equation. Empirically derived time constants are used to govern the growth, decay, and motion of the vortex. A non-dimensional time constant $\tau_{v}$ tracks the position of the vortex across the airfoil. As the vortex reaches the trailing edge the strength is allowed to decay exponentially.

The lift coefficient $C_{L}$ and drag coefficient $C_{D}$ are then calculated from resolving $C_{N}$ and $C_{C}$ into components normal and parallel to the velocity direction, and adding the minimum $\operatorname{drag} C_{D 0}$.

$$
\begin{aligned}
& C_{L}=C_{N} \cos \alpha+C_{C} \sin \alpha \\
& C_{D}=C_{N} \sin \alpha-C_{C} \cos \alpha+C_{D 0}
\end{aligned}
$$

\subsection{Gravity Loads}

The blade is subjected to gravity all the time. The component forces of gravity on each coordinate axis of the coordinate system for blade loads (see figure 3) are periodic changing by blade azimuth $\theta$. When considering the shaft tilt angle $\chi$ and the rotor coning angle $\beta$, the gravity load per unit length of the blade on each axis can be obtained as

$$
\begin{aligned}
& F_{X B g}=\bar{m} g \cos \theta \sin \beta \cos \chi \\
& F_{Y B g}=\bar{m} g \sin \theta \cos \chi \\
& F_{Z B g}=\bar{m} g \cos \theta \cos \beta \cos \chi
\end{aligned}
$$

where $\bar{m}$ is the mass per unit length of the blade, $g$ is the gravitational acceleration.

\subsection{Centrifugal Loads}

The centrifugal loads generated by the rotation of the blade are span wise. When considering the rotor coning angle $\beta$, the centrifugal loads per unit length of the blade are given by centrifugal tensile force

$$
F_{Z B C}=\bar{m} \Omega^{2} r \cos \beta
$$

and centrifugal shear force

$$
F_{X B C}=\bar{m} \Omega^{2} r \sin \beta
$$

where $\Omega$ is the rotational speed of the rotor. 
Combining the aerodynamic loads, gravity loads, and centrifugal loads, loads per unit length of the blade can be written as

$$
\begin{aligned}
& F_{X B}=F_{X B a}+F_{X B g}+F_{X B C} \\
& F_{Y B}=F_{Y B a}+F_{Y B g} \\
& F_{Z B}=F_{Z B g}+F_{Z B C}
\end{aligned}
$$

Then the distributed external time-varying loads acting on each blade element can be figured out by equation (22). Using $q(x, t)$ to denote the distributed loads, the equivalent nodal loads for each blade element are obtained as

$$
\boldsymbol{P}_{e}(t)=\int_{0}^{l} \boldsymbol{N}^{\mathrm{T}} q(x, t) d x
$$

Assembling element load vectors, the global load matrix $\boldsymbol{P}(t)$ of the blade can be obtained. Because the connection between the blade root and hub can be treated as rigid, all degrees of freedom of the blade root node are considered constrained, i.e., the displacements and rotational angles of the blade root node are set to zero.

\section{CALCULATION OF THE DYNAMIC RESPONSE}

\section{I. Modal Superposition Principle}

According to the modal superposition principle [16], the overall displacement is given by the summation of products of the mode-shape vector $\phi_{n}$ and the modal amplitude $Y_{n}$ as

$$
\boldsymbol{x}=\phi_{1} Y_{1}+\phi_{2} Y_{2}+\cdots+\phi_{N} Y_{N}=\sum_{n=1}^{N} \phi_{n} Y_{n}
$$

In matrix notation, equation (24) can be written as

$$
\boldsymbol{x}=\boldsymbol{\Phi} \boldsymbol{Y}
$$

Substituting equation (25) into equation (10) and multiplying both sides by the transpose of the $n$th mode shape vector $\phi_{n}^{\mathrm{T}}$ gives:

$$
\boldsymbol{\phi}_{n}^{T} \boldsymbol{M} \Phi \ddot{Y}(t)+\phi_{n}^{T} \boldsymbol{C} \Phi \dot{Y}(t)+\phi_{n}^{T} K \Phi \boldsymbol{Y}(t)=\phi_{n}^{T} \boldsymbol{P}(t)
$$

The undamped mode shapes are orthogonal as a result of Betti's law, so they satisfy the orthogonality conditions:

$$
\left\{\begin{array}{l}
\phi_{i}^{T} \boldsymbol{M} \phi_{j}=0 \\
\phi_{i}^{T} K \phi_{j}=0
\end{array}(i \neq j)\right.
$$

If we assume that the corresponding orthogonality condition also applies to the damping matrix, then

$$
\phi_{i}^{T} C \phi_{j}=0(i \neq j)
$$

Thus equation (26) can be written as

$$
M_{n} \ddot{Y}_{n}(t)+C_{n} \dot{Y}_{n}(t)+K_{n} Y_{n}(t)=p_{n}(t)
$$


where $M_{n}$ is the $n^{\text {th }}$ modal mass, $C_{n}$ is the $n^{\text {th }}$ modal damping, $K_{n}$ is the $n^{\text {th }}$ modal stiffness, $p_{n}(t)$ is the $n^{\text {th }}$ modal load and $p_{n}(t)=\boldsymbol{\phi}_{n}^{T} \boldsymbol{P}(t)$.

Dividing both sides of equation (29) by the modal mass $M_{n}$ gives:

$$
\ddot{Y}_{n}(t)+2 \xi_{n} \omega_{n} \dot{Y}_{n}(t)+\omega_{n}^{2} Y_{n}(t)=\frac{p_{n}(t)}{M_{n}}
$$

where $\xi_{n}$ is the $n^{\text {th }}$ modal damping ratio. The modal damping can be calculated by $C_{n}=$ $2 \xi_{n} \omega_{n} M_{n}$. Then the $N$ coupled linear damped equations of motion are converted to a set of $N$ uncoupled single degree of freedom equations of motion. When the dynamic response of each mode is obtained by resolving each uncoupled equation of motion, the displacement of the blade expressed by geometric coordinates can be obtained using equation (24). For wind turbine blade application, taking account of the former three modes is enough to meet the precision requirement, and higher modes can be neglected [24].

\subsection{Aerodynamic Damping}

Blade motion is generally resisted by two forms of viscous damping, structural and aerodynamic, which are considered in turn. Structural damping can be considered as an internal resistance opposing the rate of strain. It is related to the material of the blade and can be determined by experiments. Aerodynamic damping is mainly generated by the aerodynamic characteristics of the airfoil and depends on the rates of change of lift and drag coefficients with angle of attack. Before stall, the aerodynamic damping is positive. But when the airfoil is operating in stall, the aerodynamic damping can be negative, meaning that the flow supplies energy to the blade. This results in a potentially self-exciting system. The stallinduced vibration will occur when the energy can not be removed through structural damping, leading to damages of the blade [12]. So to adequately evaluate the aerodynamic damping and reveal its influences on the structural response is very important.

When the turbine is operating in steady condition, because the quasi-steady lift and drag coefficient curves of airfoils are pre-defined, the coefficient curve slopes on every angle of attack can be figured out in advance to be used for aerodynamic damping calculation. But when the turbine is operating in unsteady condition, because of dynamic stall, the changes of the lift and drag coefficient in magnitude and trend can not be pre-defined. Therefore the aerodynamic damping should be calculated instantaneously at each time step during the dynamic simulation.

If a blade cross section at radius $r$ experiences out-of-plane and in-plane perturbations with velocities $\dot{x}$ in the downwind direction and $\dot{y}$ in the direction opposite to that of blade rotation, as shown in figure 4, the lift and drag forces per unit length on a blade element, $L$ and $D$, can be resolved into out-of-plane and in-plane forces $F_{X}$ and $F_{Y}$, which can be written as [2]

$$
\begin{gathered}
F_{X}=\frac{1}{2} \rho c W\left[C_{L}(\Omega r-\dot{y})+C_{D}(U(1-a)-\dot{x})\right] \\
F_{Y}=\frac{1}{2} \rho c W\left[-C_{L}(U(1-a)-\dot{x})+C_{D}(\Omega r-\dot{y})\right]
\end{gathered}
$$

The aerodynamic damping coefficients per unit length for vibrations in the out-of-plane and in-plane directions are then given by

$$
\hat{c}_{X}(r)=-\frac{\partial F_{X}}{\partial \dot{x}}
$$




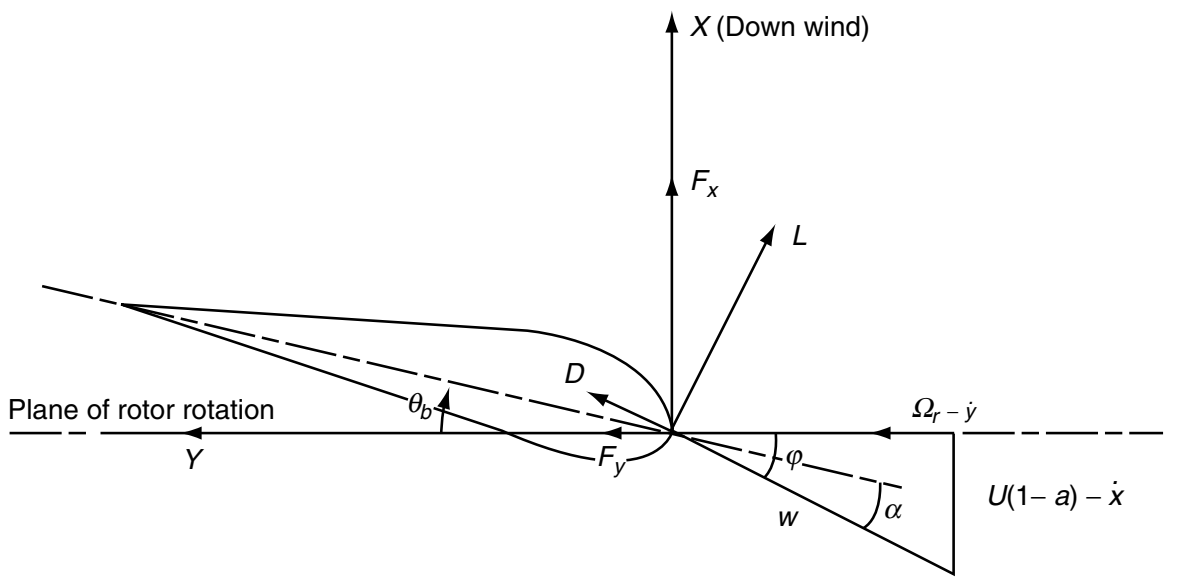

Figure 4: Velocity diagram for vibrating blade.

$$
\hat{c}_{Y}(r)=-\frac{\partial F_{\dot{Y}}}{\partial \dot{y}}
$$

Substituting $V$ for $U(1-a)$, and noting that

$$
\frac{\partial W}{\partial \dot{y}}=-\frac{\Omega r}{W}
$$

and

$$
\frac{\partial C_{L}}{\partial \dot{y}}=\frac{\partial C_{L}}{\partial \alpha} \frac{\partial \alpha}{\partial \dot{y}}=\frac{\partial C_{L}}{\partial \alpha} \frac{\partial \varphi}{\partial \dot{y}}=\frac{\partial C_{L}}{\partial \alpha} \frac{V}{W^{2}}
$$

the damping coefficients in the out-of-plane and in-plane directions are deduced as:

$$
\begin{aligned}
& \hat{c}_{X}(r)=\frac{1}{2} \rho c \frac{\Omega r}{W}\left(V C_{L}+\Omega r \frac{\partial C_{L}}{\partial \alpha}+\frac{\Omega^{2} r^{2}+2 V^{2}}{\Omega r} C_{D}+V \frac{\partial C_{D}}{\partial \alpha}\right) \\
& \hat{c}_{Y}(r)=\frac{1}{2} \rho c \frac{\Omega r}{W}\left(-V C_{L}+\frac{V^{2}}{\Omega r} \frac{\partial C_{L}}{\partial \alpha}+\frac{2 \Omega^{2} r^{2}+V^{2}}{\Omega r} C_{D}-V \frac{\partial C_{D}}{\partial \alpha}\right)
\end{aligned}
$$

Then the aerodynamic damping ratio for the $n^{\text {th }}$ mode in the out-of-plane and in-plane directions can be defined as

$$
\begin{gathered}
\xi_{X n}=\frac{\int_{0}^{R} \hat{c}_{X}(r) \phi_{n}^{2}(r) \mathrm{d} r}{2 M_{n} \omega_{n}} \\
\xi_{Y n}=\frac{\int_{0}^{R} \hat{c}_{X}(r) \phi_{n}^{2}(r) \mathrm{d} r}{2 M_{n} \omega_{n}}
\end{gathered}
$$

where $R$ is the radius of the rotor.

After getting the aerodynamic damping ratio, the overall damping ratio can be got by adding it with the structural damping ratio.

\subsection{Solution of the Equation of Motion}

The Newmark method is adopted to solve the equation of motion. According to this method, expressions for the tip displacement, velocity and acceleration at the end of each time 
step $-Y_{n 1}, \dot{Y}_{n 1}$ and $\ddot{Y}_{n 1}$ respectively-are derived in terms of the initial values $-Y_{n 0}, \dot{Y}_{n 0}$ and $\ddot{Y}_{n 0}$-as follows [25].

$$
\begin{gathered}
\ddot{Y}_{n 1}=\frac{1}{\beta h^{2}}\left(Y_{n 1}-Y_{n 0}\right)-\frac{1}{\beta h} \dot{Y}_{n 0}-\left(\frac{1}{2 \beta}-1\right) \ddot{Y}_{n 0} \\
\dot{Y}_{n 1}=\frac{\gamma}{\beta h}\left(Y_{n 1}-Y_{n 0}\right)+\left(1-\frac{\gamma}{\beta}\right) \dot{Y}_{n 0}+\left(1-\frac{\gamma}{2 \beta}\right) h \ddot{Y}_{n 0} \\
Y_{n 1}=\left\{p_{n 1}+M_{n}\left[\frac{1}{\beta h^{2}} Y_{n 0}+\frac{1}{\beta h} \dot{Y}_{n 0}+\left(\frac{1}{2 \beta}-1\right) \ddot{Y}_{n 0}\right]+C_{n}\left[\frac{\gamma}{\beta h} Y_{n 0}+\left(\frac{\gamma}{\beta}-1\right) \dot{Y}_{n 0}+\right.\right. \\
\left.\left.\left(\frac{\gamma}{2 \beta}-1\right) h \ddot{Y}_{n 0}\right]\right\} /\left(M_{n} \omega_{n}^{2}+\frac{\chi_{n}}{\beta h}+\frac{M_{n}}{\beta h^{2}}\right)
\end{gathered}
$$

where $h$ is the total duration of a time step, $\gamma$ and $\beta$ are parameters that determine the stability and the accuracy of the integration scheme. In our case $\gamma=1 / 2$ and $\beta=1 / 4$ [26] are used. The main calculation steps are:

1. Calculate the $\mathrm{n}^{\text {th }}$ modal mass $M_{n}$, modal stiffness $K_{n}$, modal damping $C_{n}$ and modal shape $\phi_{n}$ of the blade.

2. Determine the time step duration $h$. For each time step, calculate time-varying loads acting on the blade element and convert the loads into equivalent nodal loads. Then get the global load matrix $\boldsymbol{P}_{i}$. Here the suffix $i$ denotes the number of the time step.

3. For each mode, calculate the modal load $p_{n i}, p_{n i}=\boldsymbol{\phi}_{n}^{\mathrm{T}} \boldsymbol{P}_{i}$.

4. Assume initial values of the blade tip displacement, velocity and acceleration.

5. Calculate blade tip displacement, velocity and acceleration of each mode at end of first time step, using equations (41), (40) and (39) respectively.

6. Combine the responses from different modes to obtain the total response, using equation (24).

7. Repeat the above stages to obtain the blade responses at all time steps.

\section{EXAMPLE}

In the following example, the dynamic response of the rotating blade of a $1.0 \mathrm{MW}$ variable speed pitch regulated HAWT is calculated according to the method discussed above. The turbine has three blades. The length of the blade is $29.1 \mathrm{~m}$. The rated wind speed is $12 \mathrm{~m} \cdot \mathrm{s}^{-1}$.

\section{I. Numerical Results of Natural Frequencies and Mode Shapes of the Blade}

Table 1 shows the calculation result of the natural frequencies of the blade in static state and in rotating state with $\Omega=21.5 \mathrm{r} \cdot \mathrm{min}^{-1}$. As seen from table 1, the centrifugal stiffening considerably affects the natural frequency, in the out-of-plane direction the first natural frequency is increased by $7.6 \%$. For higher modes, the natural frequency increasing becomes less in percentage. In contrast, the centrifugal stiffening has less influence on the frequency in the inplane direction; the first natural frequency is only increased by $2.4 \%$. The first three mode shapes of the blade in the out-of-plane and in-plane directions are shown in figure 5 .

\subsection{Numerical Results of Aerodynamic Damping}

A power production load case of this HAWT is simulated. The working wind condition is a turbulent wind field with average wind speed $\bar{U}=12 \mathrm{~m} / \mathrm{s}$ and longitudinal turbulence intensity $\sigma=19.6 \%$. The longitudinal wind speed at hub position is shown in figure 6 . 
Table I: Natural frequencies of the blade

\begin{tabular}{ccccc}
\hline Number of modes & \multicolumn{4}{c}{ Natural Frequencies (Hz) } \\
\cline { 2 - 5 } & $\begin{array}{c}\text { Static } \\
\text { (out-of-plane) }\end{array}$ & $\begin{array}{c}\text { Rotating } \\
\text { (out-of-plane) }\end{array}$ & $\begin{array}{c}\text { Static } \\
\text { (in-plane) }\end{array}$ & $\begin{array}{c}\text { Rotating } \\
\text { (in-plane) }\end{array}$ \\
1 & 1.334 & 1.436 & 2.129 & 2.181 \\
2 & 4.014 & 4.136 & 7.454 & 7.507 \\
3 & 8.282 & 8.404 & 17.172 & 17.221
\end{tabular}

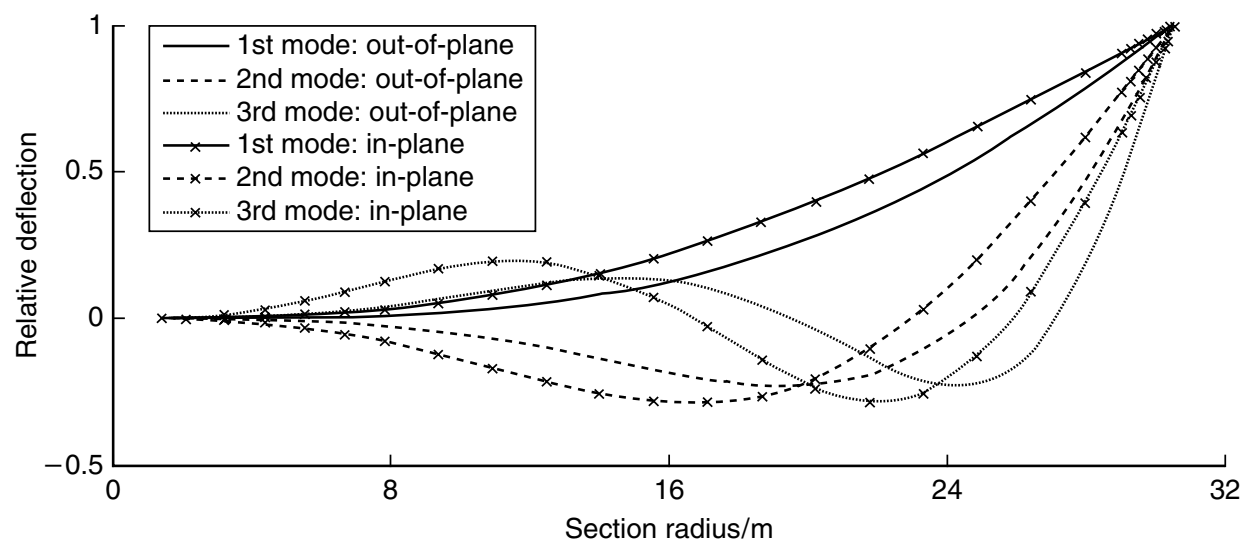

Figure 5: The former 3 mode shapes of the blade in out-of-plane and in-plane directions.

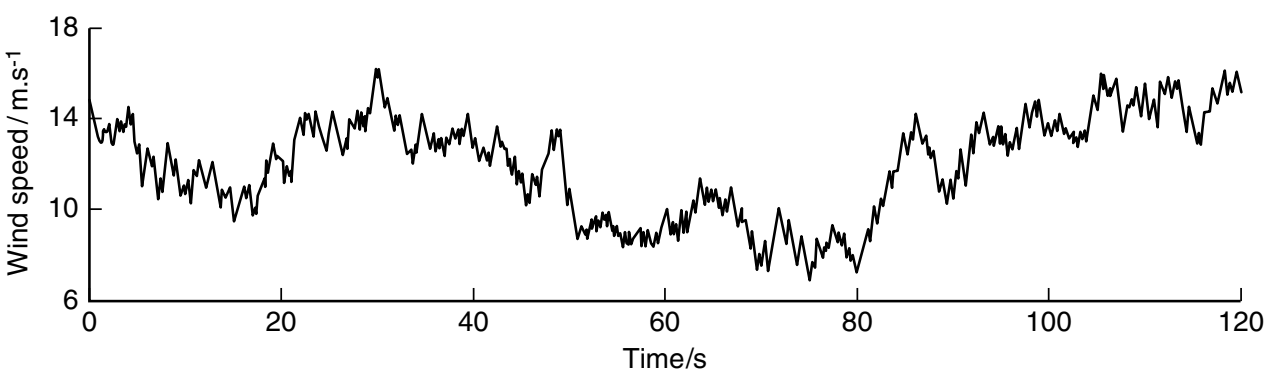

Figure 6: Longitudinal wind speed at hub center.

During this simulation, the angles of attack of blade sections change unsteadily with time because of the wind speed variation and blade pitching. According to dynamic stall, the rates of change of the lift and drag coefficients with angle of attack are no longer stationary, which causes the variation of the aerodynamic damping distribution along the blade. So during operation the aerodynamic damping coefficient of the blade is time-varying. The calculation results of the first three aerodynamic modal damping ratios in the out-of-plane and in-plane directions of this simulation are illustrated in figure 7 and figure 8 respectively.

As seen from figure 6 , for the wind speed fluctuates nearby the rated wind speed, a majority part of the blade sections is working in non-stall region. So the overall aerodynamic damping ratio of the blade maintains positive, which can restrain the vibration of the blade. As seen from figure 7 and figure 8, the first aerodynamic modal damping ratio in the out-ofplane direction is the greatest, whose maximum value is $43.5 \%$; the maximum values of the second and third aerodynamic modal damping ratios in the out-of-plane direction are $15.6 \%$ and $8.1 \%$ respectively; the aerodynamic modal damping ratio in the in-plane direction is much less and the maximum values of the former three modal damping ratios are $2.1 \%, 0.61 \%$ and $0.26 \%$ respectively. So for a HAWT, the aerodynamic damping mainly influences the structural response in the out-of-plane direction. 


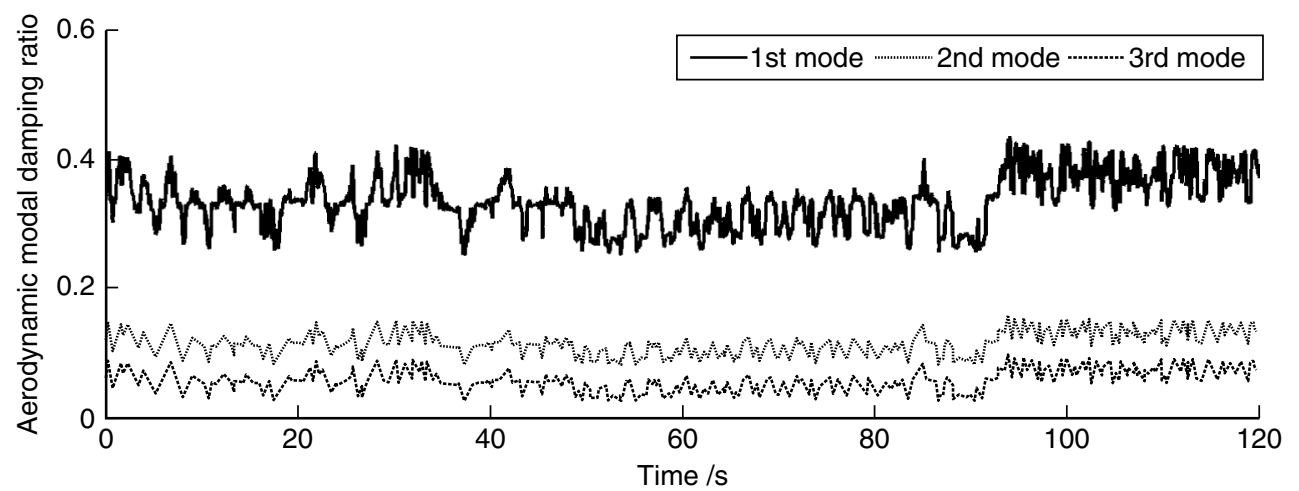

Figure 7: Time series of the aerodynamic modal damping ratio of the blade in the out-of-plane direction.

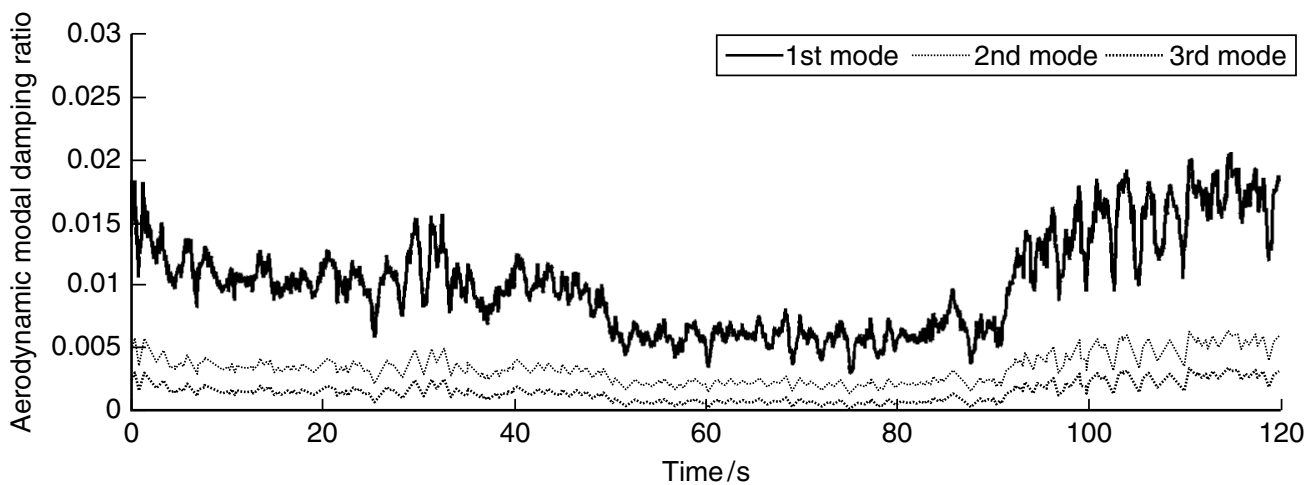

Figure 8: Time series of the aerodynamic modal damping ratio of the blade in the in-plane direction.

The former three structural modal damping ratios of blade are all selected as $0.5 \%$. The overall damping ratio is obtained by adding the structural damping ratio and the aerodynamic damping ratio. From the above calculation results, we can see that, for a rotating blade the aerodynamic damping is much greater than structural damping. So the aerodynamic damping is dominant.

\subsection{Dynamic Response of the Blade}

In this power production load case, dynamic response of the blade of the 1.0MW HAWT is calculated. The simulation duration is $120 \mathrm{~s}$, and the time step is selected as $0.05 \mathrm{~s}$. Through combining the responses from different modes, the overall blade tip displacements in the outof plane and in-plane directions are obtained, which are illustrated in figure 9. From the calculation results, we can see that, the blade of large-scale HAWT undergoes significant deflection in the out-of plane direction, even if it operates in an ordinary turbulent wind. In this case, the maximum blade tip deflection in out-of-plane direction is $2.08 \mathrm{~m}$. In contrast, the blade tip deflection in in-plane direction is much less; the maximum value is only $0.09 \mathrm{~m}$. The blade deflection in the out-of plane direction is enough to influence the aerodynamic load, structural stability and turbine security, so we should sufficiently consider the dynamic response of the blade in the wind turbine design procedure.

Figure 10 shows the influence of the centrifugal stiffening and aerodynamic damping on the blade deflection. In order to make the figure clearer, only the simulation results between 30 s and 60 s are displayed. As seen from figure 10, both the centrifugal stiffening and aerodynamic damping considerably influence the blade deflection. If considering the 

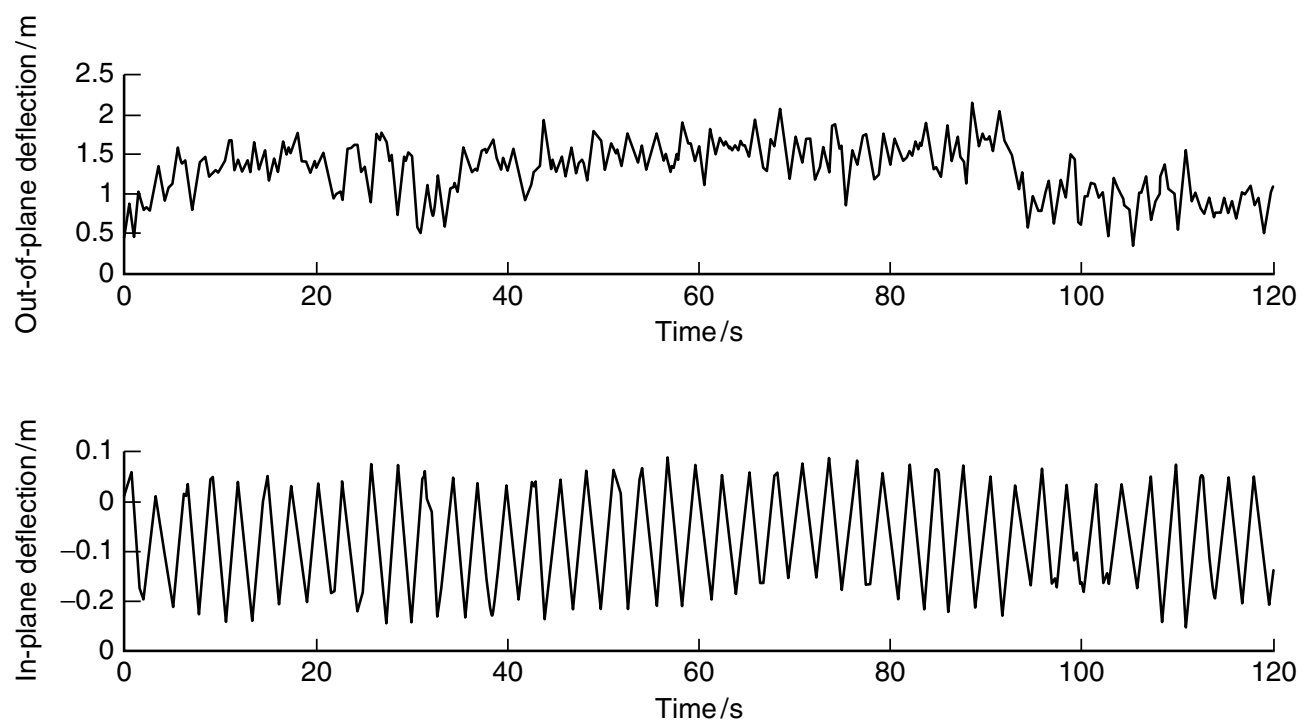

Figure 9: Blade tip deflection in the out-of-plane and in-plane directions.
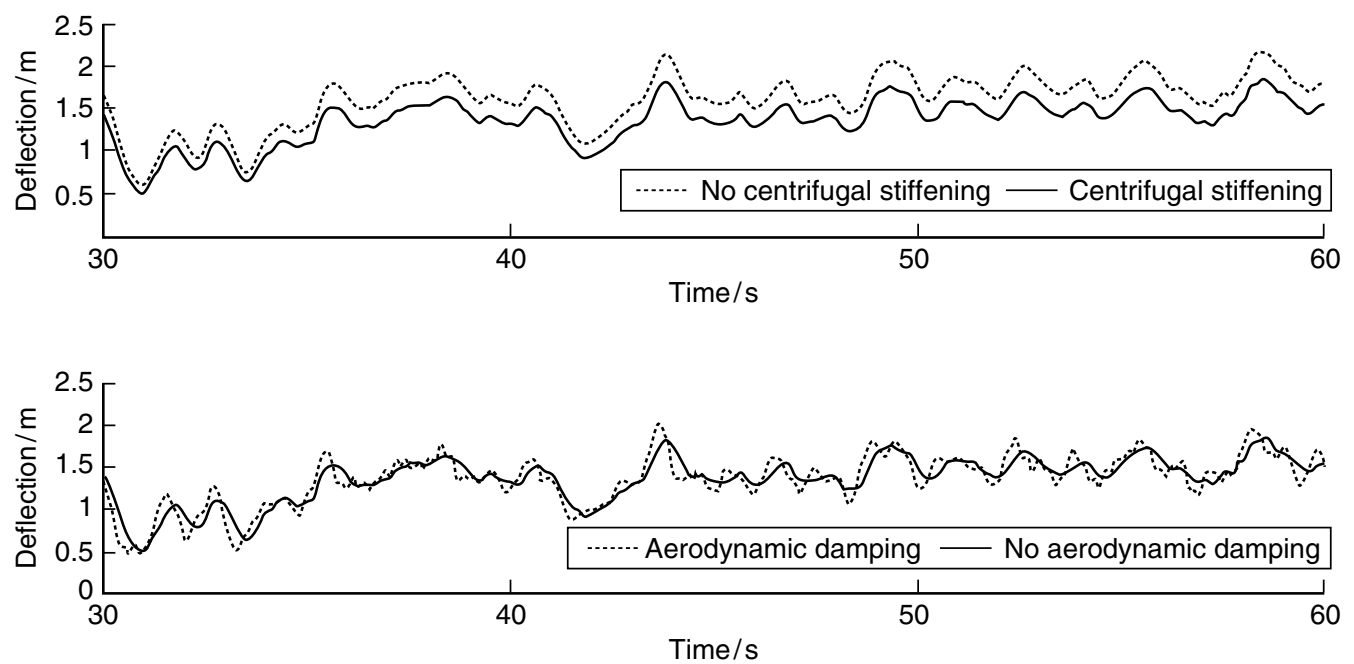

Figure 10: The influence of centrifugal stiffening and aerodynamic damping on the blade tip deflection.

centrifugal stiffening, the mean decrease of the blade tip displacement in the simulation duration is about $17.8 \%$. The aerodynamic damping not only restrains the vibration amplitude of the blade, but also reduces the vibration frequency. If considering the aerodynamic damping, the mean decrease of the blade tip vibration amplitude in the simulation duration is about $10.4 \%$. So in the wind turbine design procedure, when carrying on the load simulations, taking into account the centrifugal stiffening and aerodynamic damping is not only essential for precisely predicting the blade deformation and vibration, but also important for predicting the structural load more reasonably.

\section{CONCLUDING REMARKS}

A model for analyzing the dynamic response of the rotating blade of HAWT has been presented. The finite element dynamic equation is established and the corresponding numerical calculation method is developed. Applying the presented method, the power 
production load case of a 1.0 MW variable-speed pitch-regulated HAWT is simulated and the dynamic response of the blade is obtained. It can be concluded that:

1) Integrated with the dynamic inflow and dynamic stall model, the aerodynamic model used in this paper is capable of reflecting the transient aerodynamic loads caused by wind turbulence, yawing, rotational speed regulation and pitch regulation.

2) The structural model takes into account the centrifugal stiffening effect by introducing the geometric stiffness matrix. An aerodynamic damping model is presented and the damping ratio is calculated instantaneously during the simulation. This makes the model more reasonable to simulate the dynamic operating wind turbines and applicable for engineering application.

3) Both the centrifugal stiffening and aerodynamic damping can considerably influence the dynamic response of the blade, so they are needed to be introduced to predict the vibration, deflection and loads of the blade more precisely.

4) The flexible blades of large-scale HAWTs undergo considerable deflection and vibration during operation, which can not only influence the turbine stability, but also affect the aerodynamic loads. Thus the dynamic response of the rotating blade should be analyzed adequately when designing a wind turbine system.

\title{
ACKNOWLEDGEMENTS
}

This study was supported by the Natural Science Foundation of China (No. 50706023), the Research Program Jointly Supported by Guangdong Province and the Ministry of Education (No. 2008B090500255), the Research Start-up Foundation of Shantou University, and the Key Laboratory Foundation of Efficient \& Clean Energy Utilization, College of Hunan Province.

\author{
APPENDIX: NOMENCLATURE \\ $C \quad$ damping matrix \\ $\boldsymbol{K} \quad$ stiffness matrix \\ $\boldsymbol{M}$ mass matrix \\ $\boldsymbol{N} \quad$ hermitian interpolation function matrix \\ $\boldsymbol{N}^{\prime} \quad$ the first derivative of $\boldsymbol{N}$ with respect to $x$ \\ $\boldsymbol{P}(t) \quad$ global load matrix \\ $\boldsymbol{P}_{e}(t)$ equivalent nodal load matrix \\ $\boldsymbol{x}(t)$ displacement vector of a finite element node \\ $\Phi \quad$ structure vibration mode matrix \\ a axial flow induction factor \\ A element sectional area \\ $c \quad$ sectional chord length \\ $\hat{\mathrm{c}}(r) \quad$ aerodynamic damping coefficient per unit length \\ $C_{L} \quad$ lift coefficient \\ $C_{D} \quad$ drag coefficient \\ $C_{N} \quad$ normal force coefficient \\ $C_{N \alpha} \quad$ normal force coefficient curve slope \\ $C_{C} \quad$ chord wise force coefficient \\ $C_{T} \quad$ thrust coefficient \\ E elastic modulus
}




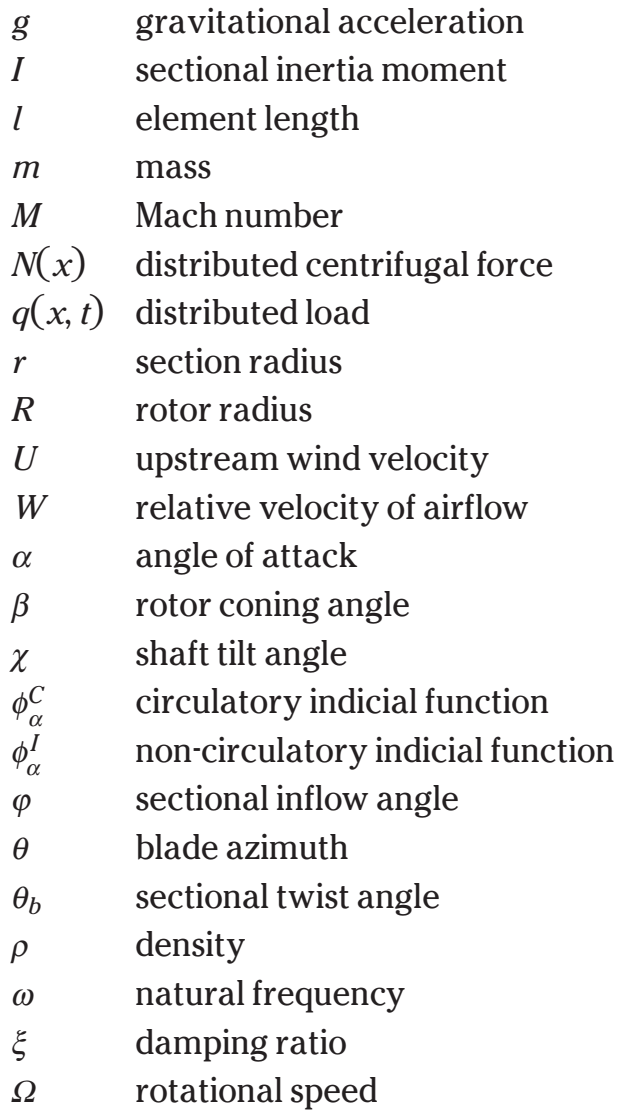

\section{REFERENCES}

[1] Quarton D C, Rasmussen F, Nath C, et al. Wind turbine design calculations - the state of the art. Proceedings of European Union Wind Energy Conference (EWEC) 1996, Sweden, 1996, 10-15.

[2] Tony Burton, David Sharpe, Nick Jenkins, et al. Wind energy handbook. New York: John Wiley \& Sons, 2005.

[3] Petersen J T. Kinematically nonlinear finite element model of a horizontal axis wind turbine. Denmark: Department of Meteorology and Wind Energy, Risø National Laboratory, 1990.

[4] Lu Ping, Qin Huifang, Luan Zhiyun. Dynamic analysis of tower structure for wind turbine based on finite element method. Chinese Journal of Mechanical Engineering, 2002, 38(9): 127-130.

[5] Li Deyuan, Liu Shengxiang, Huang Xiaohua. Numerical analysis of vortex-induced vibration of the large scale wind turbine cylindrical tower. Acta Energiae Solaris Sinica, 2008, 29(11): 1432-1437.

[6] Bossanyi E A. GH Bladed: theory manual. Bristol: Garrad Hassan \& Partners Ltd, 2007.

[7] Rachid Younsi, Ismail El-Batanony, et al. Dynamic study of a wind turbine blade with horizontal axis. European Journal of Mechanics - A/Solids, 2001, 20(2): 241-252.

[8] P J Murtagh, B Basu and B M Broderick. Mode acceleration approach for rotating wind turbine blades. Journal of Multi-Body Dynamics. 2001, 218: 241-252.

[9] Paluch B, Berlu P. A computer-aided approach of loads prediction for HAWT based on flexible multibody dynamics. Proc of 1999 EWEC. Nice, France, March 1999, 270-273. 
[10] Lee D, Hodges D H, Patil M J. Multi-flexible-body dynamic analysis of horizontal axis wind turbines. Wind Energy, 2002, (5): 281-300.

[11] Li Deyuan,Ye Zhiquan, Chen Yan. Multi-body dynamics numerical analysis of rotating blade of hotizontal axis wind turbine. Acta Energiae Solaris Sinica, 2005, 26(4): 473-481.

[12] Petersen J T, Madsen H A, Björck A, et al. Prediction of dynamic loads and induced vibrations in stall. Denmark: Risø National Laboratory, 1998.

[13] Hansen M H, Thomsen K, Fuglsang P. Two methods for estimating aeroelastic damping of operational wind turbine modes from experiments. Wind Energy, 2006, 9(2): 179-191.

[14] Larsen J W. Nonlinear dynamics of wind turbine wings. Denmark: Department of Civil Engineering, Aalborg University, 2005.

[15] Roger T. Fenner. Finite element methods for engineers. London: Imperial College Press, 1997.

[16] Clough R W, Joseph P. Dynamics of structures. New York: McGraw Hill, 1993.

[17] Murtagh P J, Basu B, Broderick B M. Along-wind response of a wind turbine tower with blade coupling subjected rotationally sampled wind loading. Engineering Structures, 2005, 27: 1209-1219.

[18] Liu Xiong, Chen Yan, Ye Zhiquan. Research on the aerodynamic performance prediction model for horizontal axis wind turbine. Acta Energiae Solaris Sinica, 2005, 26(6): 792-800.

[19] Pitt D M, Peters D A. Theoretical prediction of dynamic inflow derivatives. Vertica, 1981, 5(1): 21-34.

[20] Liu Xiong, Zhang Xianmin, Chen Yan, et al. Transient aerodynamic load prediction model for horizontal axis wind turbine based on dynamic inflow theory. Acta Energiae Solaris Sinica, 2009, 30(4): 412-419.

[21] Leishman J G, Beddoes T S. A semi-empirical model for dynamic stall. Journal of the American Helicopter Society, 1989, 34(3): 3-17.

[22] Pierce K. G. Wind Turbine Load Prediction Using the Beddoes-Leishman Model for Unsteady Aerodynamics and Dynamic Stall [D]. Utah: The University of Utah, 1996.

[23] Liu Xiong, Zhang Xianmin, Chen Yan, et al. Transient aerodynamic load prediction of horizontal axis wind turbine based on BEDDOES-LEISHMAN model. Acta Energiae Solaris Sinica, 2008, 29(12): 1449-1455.

[24] Liu Xiong, Zhang Xianmin, Chen Yan, et al. Structure dynamic response analysis of horizontal axis wind turbines. Acta Energiae Solaris Sinica, 2009, 30(6): 804-809.

[25] Zhang Xiong, Wang Tianshu. Computing structure dynamics. Beijing: Tsinghua University Press, 2003.

[26] Younsi Rachid, El-Batanony Ismail, Tritsch J B, et al. Dynamic study of a wind turbine blade with horizontal axis. Wind Energy. 2001, 20: 241-252. 
